\title{
Separación de la sínfisis púbica postparto
}

Martha Rondón-Tapia MD $^{\mathrm{a}}$ Eduardo Reyna-Villasmil MD $^{\mathrm{b}}$ Avelin Vargas-García MD $^{\mathbf{c}}$

\section{Postpartum separation of the pubic symphysis}

${ }^{a, c}$ Servicio Ginecología y Obstetricia, Hospital Central "Dr. Urquinaona". Maracaibo, Venezuela.

${ }^{b}$ Doctor en Ciencias Médicas, Servicio Ginecología y Obstetricia. Hospital Central "Dr. Urquinaona". Maracaibo, Venezuela.

\section{R E S U M E N}

La separación de la sínfisis púbica después del parto vaginal normal es rara. La etiología no es clara y se asocia con multiparidad, macrosomía, debilitamiento fisiológico de la articulación y fuerza excesiva en el área púbica. Los síntomas incluyen dolor cerca de la articulación púbica, caderas, ingle, región inferior del abdomen y parte interna de los muslos, acompañado de sensibilidad del área. Los estudios con imágenes muestran la distancia entre los huesos púbicos. El manejo médico es variable y los resultados potenciales todavía son poco comprendidos. El retraso en el diagnóstico tiene graves consecuencias para la salud de la mujer en forma aguda y a largo plazo. Se presenta una paciente de 18 años con dolor lacerante en el área púbica durante el segundo día del puerperio. Tenía antecedentes de embarazo de evolución normal y parto vaginal espontáneo, a término, con recién nacido vivo y sin complicaciones. En el examen físico había dolor moderado en la región del pubis que interfería con la marcha y los movimientos activos de ambos miembros inferiores. La radiografía pélvica anteroposterior en posición supina mostró separación anormal de la sínfisis púbica de aproximadamente 25 milímetros de extensión, sin otras anomalías óseas o congénitas. Se recomendó tratamiento conservador con recuperación a los 3 meses.

Palabras clave: sínfisis púbica; separación; pubis, embarazo.

(C) 2018 Fundación Universitaria de Ciencias de la Salud - FUCS Este es un artículo Open Access bajo la licencia CC BY-NC-ND (http://creativecommons.org/licenses/by-nc-nd/4.0/).

\section{INFORMACIÓN DEL ARTÍCULO}

Historia del artículo:

Fecha recibido: octubre 9 de 2017 Fecha aceptado: junio 29 de 2018
Autor para correspondencia. Dr. Eduardo Reyna-Villasmil sippenbauch@gmail.com
DOI

10.31260/RepertMedCir.v28.n1.2019.924 


\section{A B S T R ACT}

Pubic symphysis separation following normal vaginal delivery is uncommon. The etiology has not been fully elucidated and is associated with multiparity, macrosomia, physiological weakening of the joint or excessive force applied to the pelvic ring. Symptoms include pain around the symphysis pubis joint, hips, groin, lower abdomen and inner thigh associated with tenderness in the affected area. Imaging studies show the distance between the pubic bones. Medical management is variable and the potential results are yet poorly understood. Delay in diagnosis carries severe acute or long-term health consequences in patients. We present a case in an 18-year-old patient who complained of shearing pain in the pubic area on postnatal day 2. Past history included a normal full term pregnancy, normal spontaneous uncomplicated vaginal delivery and live-born infant. On physical examination patient had moderate pain over the pubic region associated with difficulty in walking and active leg movements. An anteroposterior radiograph of the pelvis in a supine position revealed a pubic symphyseal separation of $25 \mathrm{~mm}$, with no other bone or congenital anomalies. She was treated conservatively with complete resolution of symptoms after 3 months.

Key words: pubic symphysis; separation; pubis, pregnancy.

(C) 2018 Fundación Universitaria de Ciencias de la Salud - FUCS. This is an open access article under the CC BY-NC-ND license ( ñhttp://creativecommons.org/licenses/by-nc-nd/4.0/).

\section{N T ROD UCCIÓN}

La sínfisis púbica es una articulación de línea media, no sinovial, anfiartroidal, fibrocartilaginosa que conecta las ramas púbicas superiores. Está reforzada por cuatro ligamentos: superior, inferior, anterior y posterior. El disco cartilaginoso inter-púbico y el ligamento púbico anterior son las estructuras más importantes que mantienen la estabilidad. ${ }^{1}$

La separación de la sínfisis púbica postparto es la diástasis de la articulación, sin fractura. A menudo se asocia con dolor significativo y discapacidad durante períodos prolongados de tiempo después del parto. Puede ocurrir al final del embarazo, durante o después del parto. ${ }^{2}$ Los factores de riesgo incluyen macrosomía fetal, segunda fase del parto rápida o parto precipitado, patología pélvica previa o traumatismo del anillo pélvico, multiparidad y parto instrumental con uso de fórceps. ${ }^{3}$ Se presenta un caso de separación de la sínfisis púbica postparto.

\section{REPORTE DEL CASO}

Paciente de 18 años con antecedente de embarazo de evolución normal y parto vaginal espontáneo, a término, con recién nacido vivo masculino de 3200 gramos, sin complicaciones. Al segundo día de puerperio se quejó de dolor lacerante en el área púbica de inicio súbito, de moderada a fuerte intensidad, que empeoraba con la deambulación y mejoraba con el reposo en cúbito supino y uso de analgésicos. La paciente negaba antecedentes de enfermedades crónicas, cirugías o traumas. $\mathrm{Al}$ examen físico la paciente midió 180 centímetros y pesó 70 kilogramos. Los parámetros cardiovasculares estaban dentro de límites normales. Se encontró edema moderado de vulva sin evidencia de sangrado vaginal anormal y la episiorrafia no mostraba signos de inflamación o infección. No se observó dificultad para la micción. La palpación reveló dolor moderado en la región del pubis en la que se detectó un espacio de aproximadamente 3 centímetros entre los bordes superolaterales de los huesos púbicos. El dolor interfería con la marcha y los movimientos activos de ambos miembros inferiores. También se encontró crepitación del área púbica durante la marcha y parestesia en ambas piernas después de estar sentada o parada por periodos prolongados. La fuerza motora y los reflejos osteotendinosos profundos en ambos miembros inferiores estaban conservados.

Se consultó al servicio de ortopedia que solicitó radiografía pélvica anteroposterior en posición supina en la que se observó una separación anormal de la sínfisis púbica de aproximadamente 25 milímetros de extensión (figura 1), sin otras anomalías óseas o congénitas, por lo que se diagnosticó separación de la sínfisis púbica postparto. Debido al hallazgo radiológico y dada la estabilidad del anillo pélvico, se recomendó tratamiento conservador con reposo en cama, almohadas pélvicas, analgésicos, fisioterapia y tromboprofilaxis. El dolor disminuyó durante las siguientes seis semanas. La paciente recuperó la capacidad para deambular sin asistencia y negaba dolor durante la movilización y el descanso tres meses después del parto. Aún continúa en seguimiento ambulatorio por el servicio de ortopedia con estudios radiográficos periódicos que demuestran la reducción de la separación púbica a solo 5 milímetros después de 6 meses. 


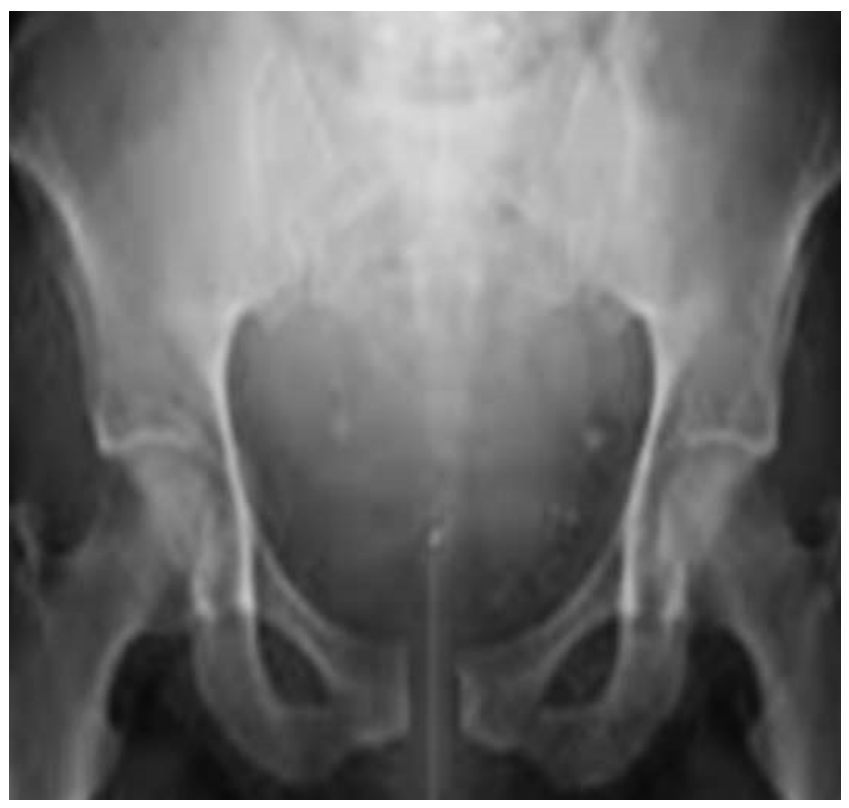

Figura 1. Radiografía pélvica anteroposterior en posición supina en la que se observa la separación de la sínfisis púbica.

\section{DISCUSIÓN}

La progesterona, la relaxina y el estrógeno hacen que los tejidos conectivos de los ligamentos de la sínfisis púbica y de las otras articulaciones se relajen durante el embarazo, permitiendo que estas respondan al estrés mecánico. La relajación de la sínfisis púbica es secundaria a cambios en la matriz extracelular que son importantes para el parto. ${ }^{4}$ Sin embargo, la relación entre concentraciones hormonales y laxitud articular durante el embarazo es poco clara, pues no se correlaciona con las concentraciones séricas de esas hormonas. ${ }^{5}$

En líneas generales, la separación de la articulación púbica no debe exceder de 8 milímetros en adultos y no embarazadas o de 10 milímetros en niños. ${ }^{6}$ Una ligera ampliación de estos valores es común durante el embarazo, llegando a un valor máximo de 10 milímetros. Este fenómeno es más común en las multíparas que en las primigestas. Por lo tanto, la separación de 11 a 13 milímetros es diagnóstica y representa una subluxación. Cuando es superior a 14 milímetros puede indicar daño de la articulación. $^{\text {? }}$

La separación de la sínfisis púbica es una complicación del embarazo (puede ocurrir durante el parto, en especial en el instrumental, al final del embarazo o durante el puerperio) en la cual la sínfisis púbica se separa. Produce dolor pélvico agudo que puede conducir a complicaciones severas a largo plazo que pasan sub-diagnósticadas ${ }^{8}$. La incidencia varía entre 1 de 300 a 1 de $30.000 .^{8,9}$ Sin embargo, la frecuencia parece estar disminuyendo, ya que los partos vaginales difíciles e instrumentales son cada vez más escasos. ${ }^{10}$ La frecuencia varía ocurriendo en $74 \%$ de los casos en primigestas, $12 \%$ en el primer trimestre, $34 \%$ en el segundo y $52 \%$ en el tercero. ${ }^{4}$ Las pacientes con esta afección por lo general tienen un buen pronóstico, debido a que el parto también es la cura. La lesión es parcialmente estable y se clasifica como libro abierto, ya que desde el punto de vista rotacional son inestables por el compromiso de los ligamentos anteriores, pero son estables en sentido vertical debido a la integridad del ligamento posterior. ${ }^{1}$ En ausencia de manejo adecuado la separación de la sínfisis púbica puede producir dolor crónico. También se asocia con incontinencia, dispareunia y/o discapacidad crónica. ${ }^{2}$ Es incierto qué porcentaje de estas pacientes presentará complicaciones a largo plazo.

Su etiología aún es desconocida. Varios factores de riesgo han sido relacionados incluyendo parto laborioso o precipitado, desproporción cefalopélvica, macrosomía fetal, distocia de hombroso presentacionesanómalas, multiparidad, traumatismos pélvicos previos y fuerza excesiva externa aplicada la pelvis. También se ha mencionado la abducción excesiva de los muslos durante el parto, parto vaginal instrumental, anomalías preexistentes debidas a displasia congénita, osteomalacia, condromalacia, raquitismo, tuberculosis, artritis y excesivo reblandecimiento de los ligamentos por efecto hormonal., ${ }^{3,10}$

El diagnóstico se realiza basados en la clínica y los hallazgos radiográficos. La paciente presenta dolor agudo e intenso en la región púbica que se puede extender hacia región lumbar y miembros inferiores, el cual casi siempre se agrava con el movimiento. Todo esto puede estar acompañado de crepitación al caminar. ${ }^{3}$ En la mayoría de las pacientes el dolor desaparece en los primeros 6 meses después del parto, con solo $25 \%$ de los casos presentándolo a los 4 meses y solo un pequeño porcentaje después de 12 meses. Las excepciones ocurren cuando el parto es instrumental, entre 1 y $7 \%$ de los casos. ${ }^{1,4}$ En el examen físico se puede apreciar dolor y deformidad de la zona púbica, alteraciones de la movilidad de los miembros inferiores y en ocasiones disfunción vesical. La palpación de la sínfisis púbica puede mostrar un espacio en la articulación, junto con edema del tejido blando que lo recubre y el examen vaginal puede revelar separación palpable de la sínfisis. ${ }^{3}$

El diagnóstico de separación de la sínfisis púbica se confirma con estudios de imagen. En las radiografías se observa separación ancha anormal entre los huesos púbicos, como en el presente caso. Se puede demostrar mayor inestabilidad en radiografías de pie en posición de flamenco. Un desplazamiento vertical superior de 10 milímetros indicaría inestabilidad de la sínfisis púbica, aquellas mayores de 20 milímetros se asocian con lesión sacroiliaca. ${ }^{9}$ La tomografía computarizada y la resonancia magnética suministran información detallada de la sínfisis púbica y las articulaciones sacroilíacas. Esta última es de mayor utilidad al demostrar la lesión de tejidos blandos e inflamación subcondral-ósea. ${ }^{5}$ 
En los casos de diáfisis de la sínfisis púbica se recomienda tratamiento conservador (incluyendo analgesia, restricción de actividad, reposo en posición decúbito lateral, uniones pélvicas apropiadamente ajustadas, dispositivos de ambulación y terapia física), con lo cual suelen aliviarse los síntomas en pocos días y se logra recuperación funcional completa en 4 a 8 semanas. ${ }^{9,10}$ El tratamiento quirúrgico a veces es necesario en aquellas pacientes con separaciones mayores de 40 milímetros y cuando se observa dolor persistente, reducción inadecuada con tratamiento conservador, separación recurrente, inestabilidad del anillo pélvico o lesión cutánea. La fijación externa o interna de la rama púbica superior es el tratamiento de elección para mantener la estabilidad mientras los ligamentos cicatrizan. Sin embargo, los siguientes embarazos tienen que ser resueltos por vía alta. Otra desventaja del tratamiento quirúrgico es que interfiere con la lactancia por el uso de analgésicos, antibióticos y profilaxis tromboembólica. ${ }^{6}$ Debido a lo infrecuente de esta condición y la limitada evidencia disponible, la terapia adecuada de la separación de la sínfisis púbica sigue siendo controversial. $^{7}$

\section{CONCLUSIÓN}

Se debe sospechar la separación púbica cuando la paciente se queja de dolor agudo y persistente en el área pélvica después del parto. El tratamiento puede ser conservador o quirúrgico. Se ha propuesto el tratamiento quirúrgico de la pelvis inestable postparto cuando el método conservador no ha podido controlar el dolor intenso.

\section{REFEREN CIAS}

1. Becker I, Woodley SJ, Stringer MD. The adult human pubic symphysis: a systematic review. J Anat. 2010;217:475-87. doi: 10.1111/j.1469-7580.2010.01300.x.

2. Herren C, Sobottke R, Dadgar A, Ringe MJ, Graf M, Keller K, Eysel P, Mallmann P, Siewe J.. Peripartum pubic symphysis separation-Current strategies in diagnosis and therapy and presentation of two cases. Injury. 2015;46:1074-80. doi: 10.1016/j.injury.2015.02.030.

3. Moiety FM, Azzam AZ. Fundal pressure during the second stage of labor in a tertiary obstetric center: a prospective analysis. J Obstet Gynaecol Res. 2014;40:946-53. doi: 10.1111/jog.12284.

4. Hashem G, Zhang Q, Hayami T, Chen J, Wang W, Kapila S. Relaxin and beta-estradiol modulate targeted matrix degradation in specific synovial joint fibrocartilages: progesterone prevents matrix loss. Arthritis Res Ther. 2006;8:R98.
5. Zou Y, Fan F, Ma A, Yue Y, Mao W, Ma X. Hormonal changes and somatopsychologic manifestations in the first trimester of pregnancy and post partum. Int J Gynaecol Obstet. 2009;105:469. doi: 10.1016/j.ijgo.2008.12.001.

6. Mathews R, Gearhart JP, Bhatnagar R, Sponseller P. Staged pelvic closure of extreme pubic diastasis in the exstrophy-epispadias complex. J Urol. 2006;176:2196-8.

7. Aydın S, Bakar RZ, Aydın ÇA, Özcan P. Assessment of postpartum symphysis pubis distention with 3D ultrasonography: a novel method. Clin Imaging. 2016;40:185-90. doi: 10.1016/j. clinimag.2015.10.015.

8. Yoo JJ, Ha YC, Lee YK, Hong JS, Kang BJ, Koo KH. Incidence and risk factors of symptomatic peripartum diastasis of pubic symphysis. J Korean Med Sci. 2014;29:281-6. doi: 10.3346/jkms.2014.29.2.281.

9. Budak MJ, Oliver TB. There's a hole in my symphysis -- a review of disorders causing widening, erosion, and destruction of the symphysis pubis. Clin Radiol. 2013;68:173-80. doi: 10.1016/j. crad.2012.03.021.

10. Hierholzer C, Ali A, Toro-Arbelaez JB, Suk M, Helfet DL. Traumatic disruption of pubis symphysis with accompanying posterior pelvic injury after natural childbirth. Am J Orthop (Belle Mead NJ). 2007;36:E167-7 